\title{
NOTE
}

\section{Lactococcus garvieae and Streptococcus iniae infections in rainbow trout Oncorhynchus mykiss: similar, but different diseases}

\author{
A. Eldar ${ }^{1}$, C. Ghittino ${ }^{2, *}$ \\ 'Department of Poultry and Fish Diseases, Kimron Veterinary Institute, POB 12, 50250 Bet-Dagan, Israel \\ ${ }^{2}$ Fish Disease Laboratory, IZS - State Veterinary Institute, Via Bologna 148, I-10154 Turin, Italy
}

\begin{abstract}
Clinical and macroscopic findings (anorexia, lethargy, loss of orientation and exophthalmia) indicate that Streptococcus iniae and Lactococcus garvieae infections of trout share some common features, but histopathology reveals notable differences between the 2 diseases. Meningitis and panophthalmitis are the main lesions among $S$. iniae infected trout, whereas $L$. garvieae infection results in a hyperacute systemic disease. Differences in the $\mathrm{LD}_{50} \mathrm{~S}$ of the 2 pathogens and the sudden onset of signs and death correlate with the histopathological findings, indicating the severity of $L$. garvieae infection of trout.
\end{abstract}

KEY WORDS: Trout - Streptococcus iniae - Lactococcus garvieae Pathology. Experimental disease

During the last decade, Gram-positive cocci have become important fish pathogens. Epidemic and sporadic diseases have been reported from different parts of the world, notably Japan (Kitao 1993), Singapore (Foo et al. 1985), Australia (Carson et al. 1993), Israel (Eldar et al. 1995), Italy (Ghittino \& Prearo 1992), Spain (Toranzo et al. 1995, Domenech et al. 1996), France (Michel et al. 1997), South Africa (Braag \& Broere 1986) and the United States (Perera et al. 1994). Amended taxonomy reveals that at least 6 different species of Gram-positive cocci, including streptococci (Pier \& Madin 1976, Domenech et al. 1996), lactococci (Collins et al. 1984, Williams et al. 1990, Domenech et al. 1993, Eldar et al. 1996), and vagococci (Wallbanks et al. 1990), are responsible for such conditions. Despite progress in taxonomy and diagnosis (Zlotkin et al. $1998 \mathrm{a}, \mathrm{b})$, the pathological basis of the diseases has not been addressed. Infections with Gram-positive cocci are followed by non-specific lesions, such as

\footnotetext{
·Addressee for correspondence. E-mail: izstoghi@ipsnet.it
}

haemorrhage, ophthalmitis and congestion (Kusuda et al. 1991, Domenech et al. 1996). Common signs (lethargy, dark pigmentation, erratic swimming and exophthalmos with clouding of the cornea) are also present in Lactococcus garvieae (Collins et al. 1984; junior synonym: Enterococcus seriolicida (Kusuda et al. 1991, Domenech et al. 1993, Eldar et al. 19961) and Streptococcus iniae (Pier \& Madin 1976) infections of rainbow trout Oncorhynchus mykiss reared above $15^{\circ} \mathrm{C}$. Our findings now show that these are 2 defined conditions. L. garvieae infection of trout produces a generalized disease and rapid death, while the disease induced by $S$. iniae results in a more prolonged course with specific lesions.

Materials and methods. Sampling procedure for pathology and bacteriological analysis: Specimens were collected from 2 farms. Farm 1, which has a history of production losses due to Lactococcus garvieae infection, is located in Northern Italy (Po Valley); at the time of sampling the water temperature was $18^{\circ} \mathrm{C}$. Farm 2 is located in Northern Israel (Upper Galilee) and is supplied by water at a constant temperature of $16^{\circ} \mathrm{C}$; this farm has a similar history of Streptococcus iniae infection. At the time of collection both farms were experiencing heavy mortality, and on bacteriological examination (20 fish from each farm), pure cultures of Gram-positive cocci were identified as $S$. iniae or L. garvieae (Eldar et al. 1995, 1996) when grown on blood agar. Tissues (eye, brain, heart, spleen, kidney, liver, intestine and muscle) from 15 diseased rainbow trout were fixed in 10\% neutral buffered formalin and stained by haematoxylin and eosin (HE). Gross lesions were recorded.

Fish and experimental procedures: Groups of 50 rainbow trout ( $100 \mathrm{~g}$ each) were maintained at $16^{\circ} \mathrm{C}$ in $300 \mathrm{l}$ tanks supplied with constant aeration and continuous water flow. Field isolates, Lactococcus garvieae 
Table 1 Streptococcus iniae and Lactococcus garvieae infecting Oncorhynchus mykiss. Gross pathology of naturally infected trout. Values show number of fish of 20 specimens showing symptom

\begin{tabular}{|lcc|}
\hline Pathology & $\begin{array}{c}\text { S. iniae } \\
\text { infected trout }\end{array}$ & $\begin{array}{c}\text { L. garvieae } \\
\text { infected trout }\end{array}$ \\
\hline Darkening of the skin & $16 / 20$ & $20 / 20$ \\
Lethargy & $20 / 20$ & $20 / 20$ \\
Intracranial oedema & $17 / 20$ & $18 / 20$ \\
Protrusion of the eye & $17 / 20$ & $19 / 20$ \\
Clouding of the cornea & $17 / 20$ & $12 / 20$ \\
Intraocular haemorrhage & $5 / 20$ & $2 / 20$ \\
Diarrhea & $2 / 20$ & $20 / 20$ \\
Gill pallor & $7 / 20$ & $2 / 20$ \\
External haemorrhage & $0 / 20$ & $10 / 20$ \\
Internal haemorrhage & $2 / 20$ & $18 / 20$ \\
Anus inflammation & $2 / 20$ & $14 / 20$ \\
Splenomegaly & $5 / 20$ & $20 / 20$ \\
Hepatic lesions & $0 / 20$ & $15 / 20$ \\
Congested kidneys & $0 / 20$ & $18 / 20$ \\
Dilated intestine & $2 / 20$ & $20 / 20$ \\
Pericardial lesions & $0 / 20$ & $15 / 20$ \\
\hline
\end{tabular}

ITP 2001 and Streptococcus iniae Dan-1, were grown at $24^{\circ} \mathrm{C}$ on Columbia agar base (Difco) supplemented with $5 \%$ defibrinated sheep blood. After $24 \mathrm{~h}$ the colonies were scraped off, washed twice in PBS (pH 7.2) and resuspended in the same buffer at various concentrations, ranging from $1.25 \times 10^{2}$ to $2.5 \times 10^{8} \mathrm{CFU} \mathrm{ml} \mathrm{m}^{-1}$. Each group of fish was infected by intraperitoneal injection of $100 \mu \mathrm{l}$ of bacterial suspension. Mortality was monitored until no more dead fish were observed for $2 \mathrm{wk}$. Positive bacterial culture from dead fish was the criterion for specific mortality.

Results. Pathology: Macroscopic findings could differentiate between the 2 diseases (Table 1). The Streptococcus iniae infected fish were found with ocular abnormalities (clouding of cornea and exophthalmos) and intracranial oedema. The Lactococcus garvieae infected trout presented, in addition to the signs described above, haemorrhage, congestion of the internal organs and enteritis. Histologically, both L. garvieae and $S$. iniae infected trout showed acute meningitis, consisting of an exudate covering the brain surface. Within the exudate, colonies of Gram-positive cocci were widely distributed over the meningeal surface and within the Virchoff's spaces (Fig. 1). These findings correlate with clinical findings of lethargy and loss of orientation.

Ocular alterations resulted from a severe haemorrhagic panophthalmitis with destruction of the anterior and posterior chambers; the optic nerve papilla was heavily affected and inflammation progressed into the retrobulbar fat and striated muscle.

These findings were identical in grading and distribution in both infections, contrasting with the following histological differences:

(1) Serositis, sometimes extending to the myocardium, was present in the Lactococcus garvieae infected fish (Fig. 2), whereas no similar lesion was detected among Streptococcus iniae infected trout.

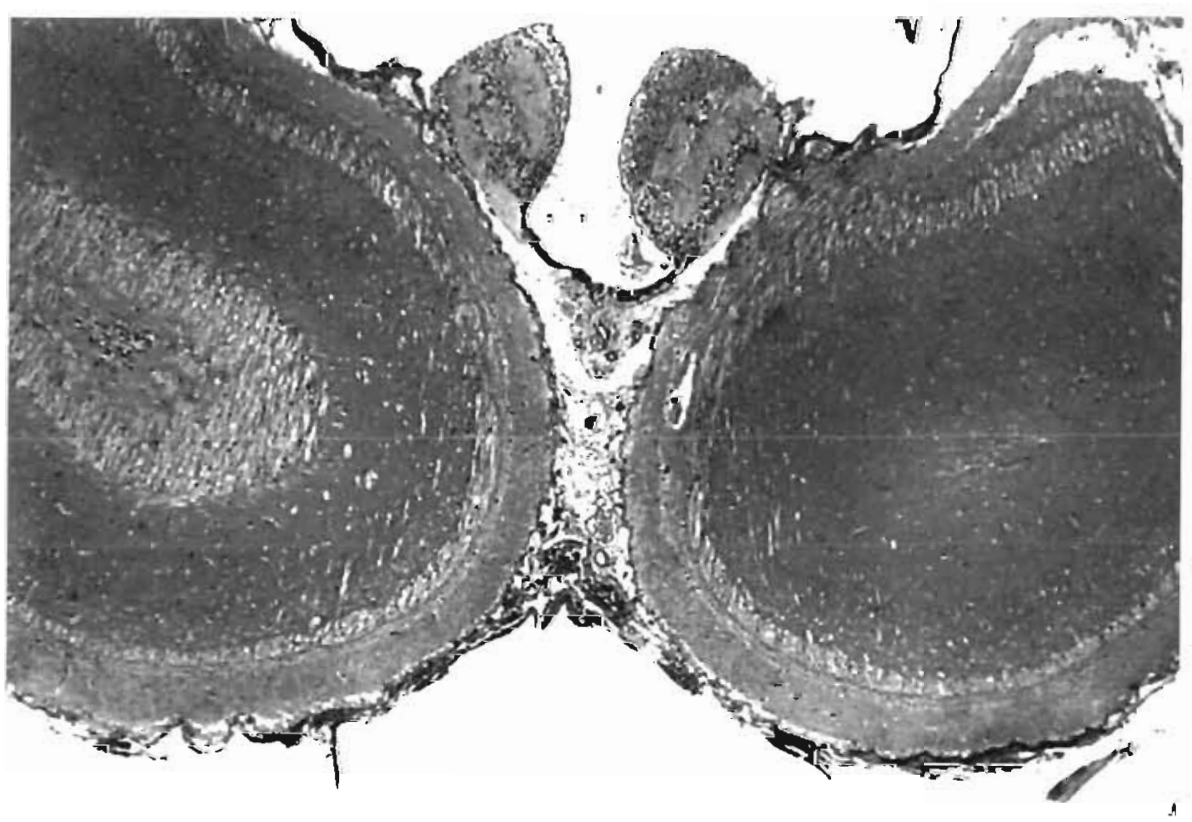

Fig. 1. Streptococcus iniae infected trout brain. Acute meningitis consistent with fibrino-purulent exudate widely distributed over the meningeal surface and within the Virchoff's spaces. The brains in trout infected by Lactococcus garvieae show a similar picture. $\mathrm{HE} \times 20$ 


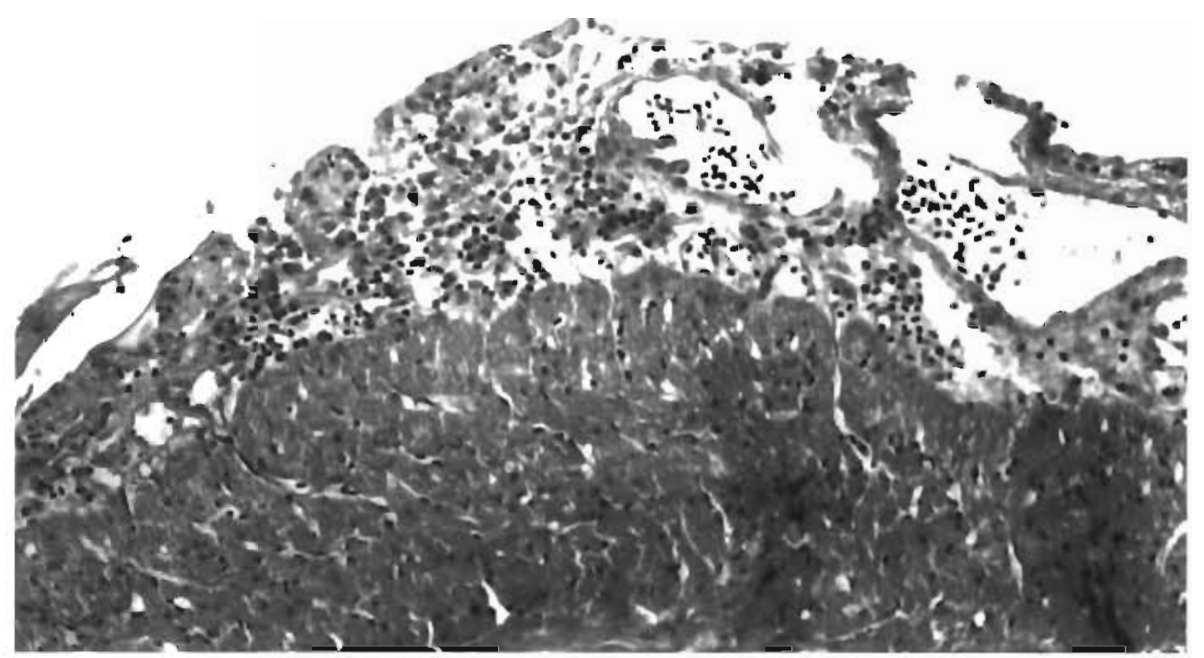

Fig. 2. Lactococcus garvieae infected trout heart. Moderate pericarditis; note mesothelial cell proliferation and fat tissue depletion. HE $\times 200$

The most notable difference was found in the celomic cavity. The L. garvieae infected fish suffered a severe peritonitis with fat necrosis (Fig. 3), whereas $S$. iniae infected trout had a mild peritonitis, according to the degree of pericardial involvement.

(2) Intestine. The architecture of the mucosal layer was preserved in the Streptococcus iniae infected trout, with unremarkable crypt density and distribution. A mild acute inflammatory infiltrate was seen over the mesothelial peritoneal layer. In contrast, Lactococcus garvieae infected fish had extensive superficial erosions with pseudomembrane-like formation (Fig. 3).

(3) Liver. In both infections, portal spaces revealed unremarkable bile ducts, blood vessels and limiting

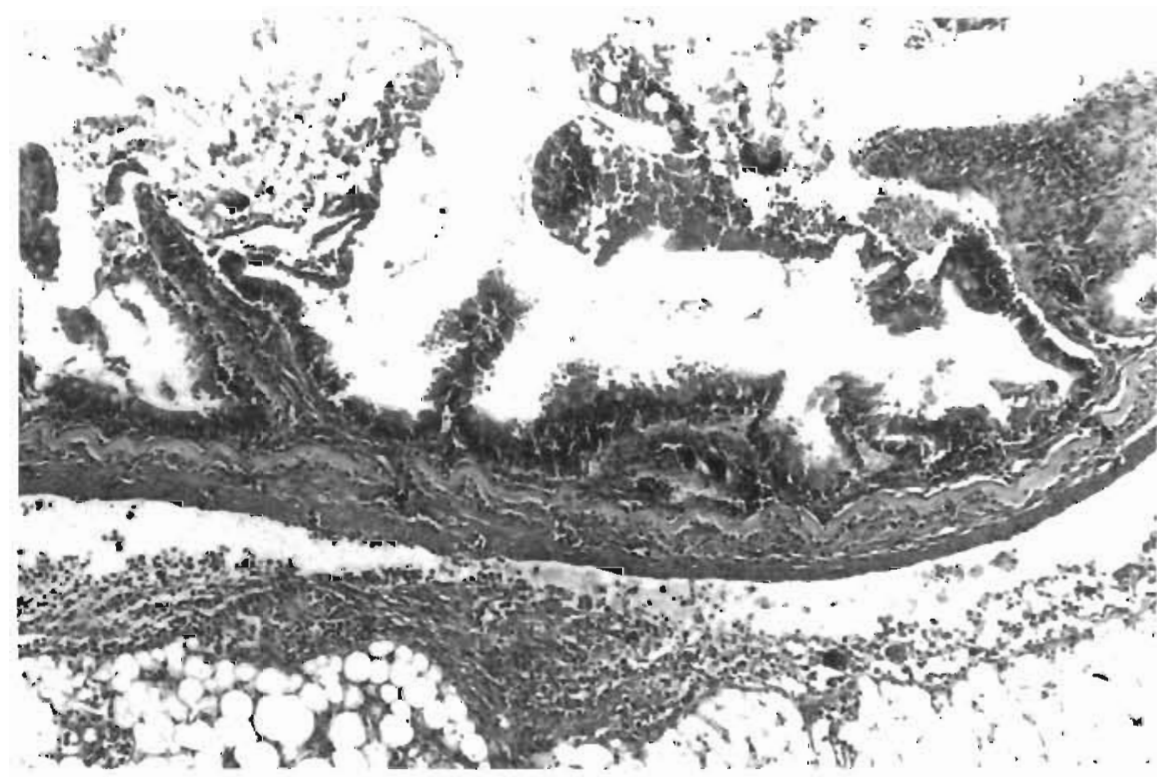

Fig. 3. Lactococcus garvieae infected trout intestine. Severe peritonitis with fat necrosis; extensive superficial erosions with pseudomembrane-like formation and globet cell depletion in the mucosa. HE $\times 100$ 


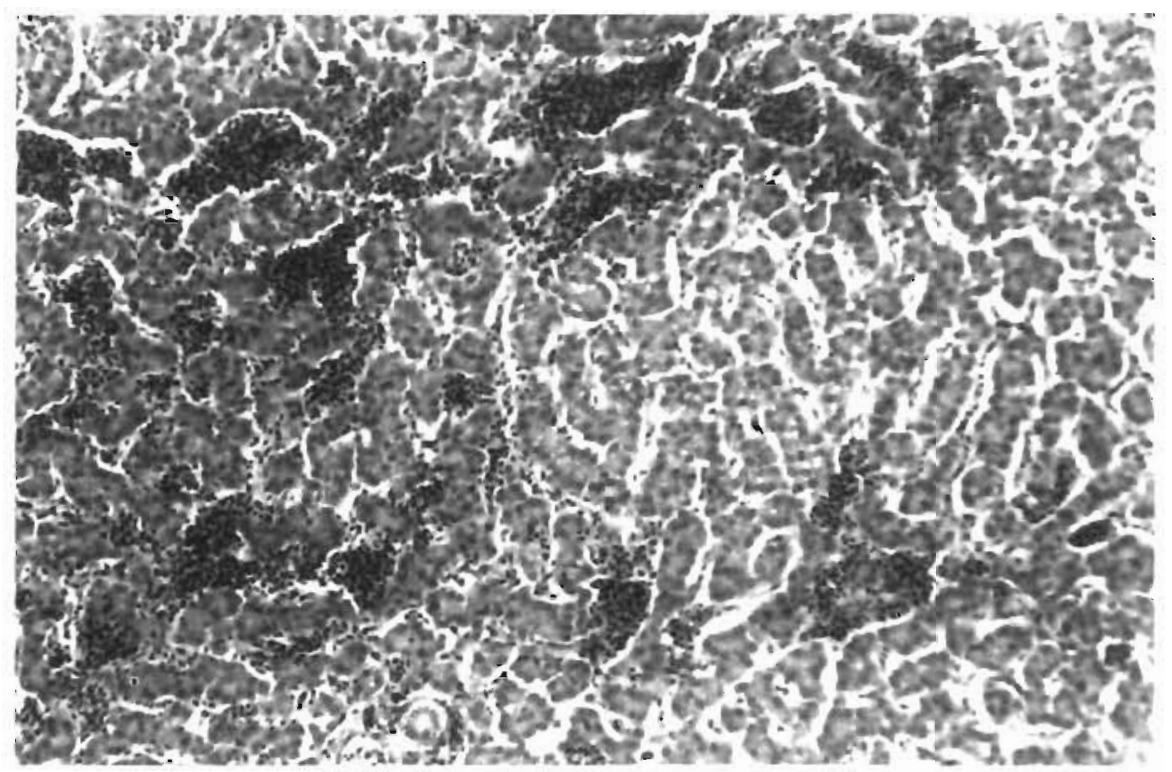

Fig. 4, Lactococcus garvieae infected trout liver. Peliosis Hepatis. Note RBC lakes within liver parenchyma. HE $\times 100$

plates. A notable contrast was found in the parenchyma of trout infected by the different bacteria. Blood-filling spaces in a diffuse fashion were a constant feature of Lactococcus garvieae infection, consistent with Peliosis Hepatis (Fig. 4). Hepatic tissue of Streptococcus iniae infected trout was unremarkable.

(4) Kidney. The glomeruli had a well preserved urinary space, with no adhesions or crescent-like structures. No hypercellularity or capillary wall thickening was seen. Tubular structure and cellularity were normal in Streptococcus iniae infected fish, whereas a marked reactive hyperplasia of the haematopoietic tissue was evident in Lactococcus garvieae infected fish (Fig. 5).

Experimental infections: The $\mathrm{LD}_{50}$ of Streptococcus iniae was $2.5 \times 10^{5} \mathrm{CFU}_{\text {fish }}^{-1}$ at $21 \mathrm{~d}$ post challenge, while that of Lactococcus garvieae was $1.25 \times 10^{1} \mathrm{CFU}$ fish $^{-1}$, attained $7 \mathrm{~d}$ post challenge. The kinetics of the 2 experimental diseases varied in: (1) the lag period (the time required for the death of the second fish), which was $8 \mathrm{~d}$ for $S$. iniae, but only $3 \mathrm{~d}$ for $L$. garvieae, and (2) the average day of death (defined as the time

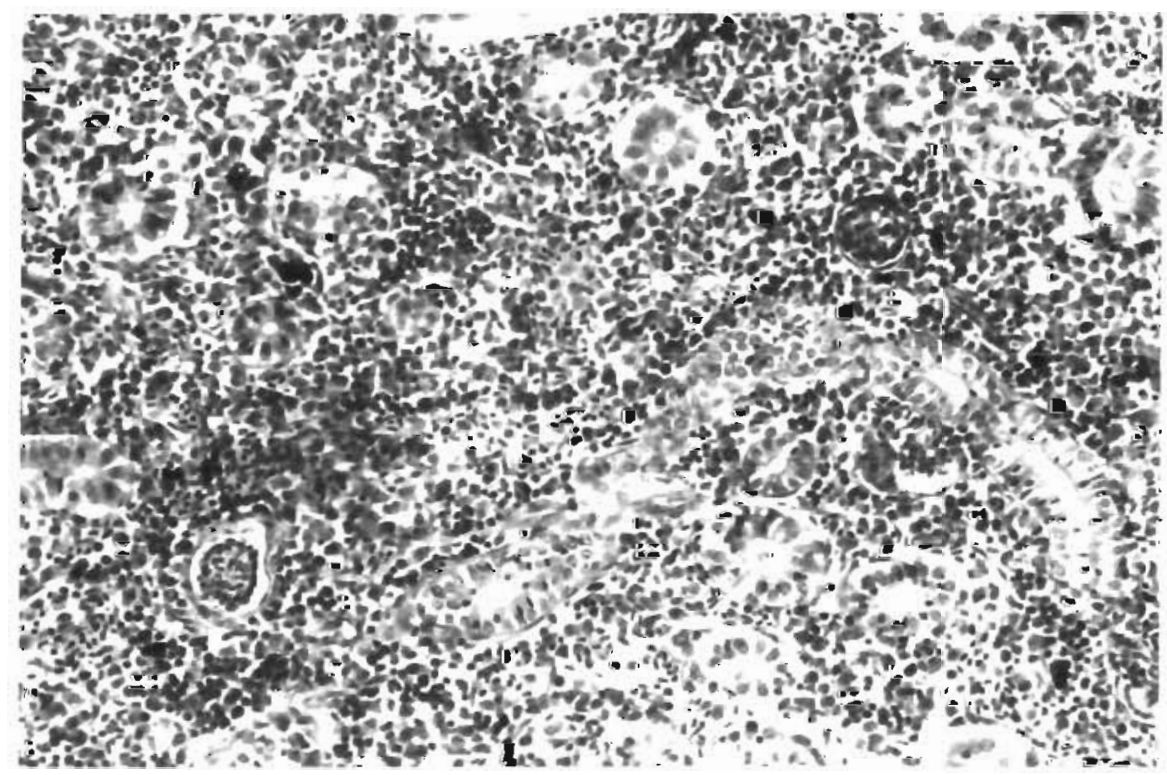

Fig. 5. Lactococcus garvieae infected trout kidney. Marked reactive hyperplasia of the haematopoietic tissue, expressed as loss of the normal tubule distribution. $\mathrm{HE} \times 200$ 
required for the death of $25 \%$ of the subjects after the first death), which was determined to be $2 \mathrm{~d}$ for $L$. garvieae and $5 \mathrm{~d}$ for $S$. iniae.

Discussion. Since clinical signs are similar in both cases, comparable pathological findings are expected to be found within the visceral cavity. Interestingly, despite common findings (meningitis and panophthalmitis), pathological differences in internal organs enabled us to differentiate between the 2 diseases. This finding was termed as 'oculo-splancnic dissociation', consistent with a severe serositis restricted to Lactococcus garvieae infected fish. The macroscopic identifiable peritonitis and the disease produced by $L$. garvieae infection provided an important clue as to the nature of the aetiological agent. The short interval between induction of the experimental disease and death, along with the differences in the $\mathrm{LD}_{50} \mathrm{~S}$, support the clinical and pathological data, demonstrating the severity of $L$. garvieae infection.

The hyperacute disease following Lactococcus garvieae infection in trout (which resembles the disease caused by the same agent in yellowtail Seriola quinqeradiata; Kusuda 1991) and the acute disease of trout resulting from Streptococcus iniae infection are notably different from the disease caused by Vagococcus salmoninarum, the third taxon of Gram-positive cocci involved in diseases of salmonids (Michel et al. 1997). $V$, salmoninarum mainly infects broodstock fish held in cold water, producing hyperaemia, and chronic disease characterized by tegumentary lesions and a proliferative response of the cardiovascular system. Neither tegumentary lesions nor multifocal vascular alterations typical of $V$. salmoninarum infected trout were observed.

Whilst bacteriological culture and biochemical identification of the casual agent remain the ultimate confirmation of each of these diseases, the data generated in the present work are of practical use for fish pathologists who investigate trout mortality resulting from Gram-positive coccal infections.

Acknowledgements. We would like to thank Dr M. Prearo and Dr A. Hurvitz for their technical assistance. This work was supported by a joint American-Israeli grant (BARD IS2727-96R)

\section{LITERATURE CITED}

Braag RR, Broere JSE (1986) Streptococcosis in rainbow trout in South Africa. Bull Eur Assoc Fish Pathol 6:89-91

Carson J, Gudkovs N, Austín B (1993) Characteristics of an Enterococcus-like bacterium from Australia and South Africa, pathogenic for rainbow trout (Oncorhynchus mykiss, Walbaum). J Fish Dis 16:381-388

Editorial responsibility: David Bruno,

Aberdeen, Scotland, UK
Collins MD, Farrow FAE, Phillips BA, Kandler O (1984) Streptococcus garvieae sp. nov. and Streptococcus plantarum sp. nov. J Gen Microbiol 129:3427-3431

Domenech A, Prieta J, Fernandez-Garayzabal JF, Collins MD, Jones D, Dominguez L (1993) Phenotypic and phylogenetic evidence for a close relationship between Lactococcus garvieae and Enterococcus seriolicida. Microbiologia SEM 9:63-68

Domenech A, Fernandez-Garayzabal JF, Pasqual C, Garcia JA, Cutuli MT, Moreno MA, Collins MD, Dominguez L (1996) Streptococcosis in cultured turbot, Scophthalmus maximus (L.), associated with Streptococcus parauberis. J Fish Dis 19:33-38

Eldar A, Frelier PF, Asanta L, Varner PW, Lawhon S, Bercovier H (1995) Streptococcus shiloi, the name for an agent causing septicemic infection in fish, is a junior synonym of Streptococcus iniae. Int J Syst Bacteriol 45: $840-842$

Eldar A, Ghittino C, Asanta L, Bozzetta E, Goria M, Prearo M, Bercovier H (1996) Enterococcus seriolicida is a junior synonym of Lactococcus garvieae, a causative agent of septicemia and meningoencephalitis in fish. Curr Microbiol 32:85-88

Foo JTW, Ho B, Lam TJ (1985) Mass mortality in Siganus canaliculatus due to streptococcal infection. Aquaculture 49:185-195

Ghittino C. Prearo M (1992) Report of streptococcosis in rainbow trout (Oncorhynchus mykiss) in Italy: preliminary note. Soc It Patol Ittica Bull 8:4-11

Kitao T (1993) Streptococcal infections. In: Inglis $V$, Roberts RJ, Bromage NR (eds) Bacterial disease of fish. Blackwell Scientific Publications, Oxford, p 196-210

Kusuda K, Kawai K, Salati F, Banner CR, Fryer JL (1991) Enterococcus seriolicida sp. nov., a fish pathogen. Int J Syst Bacteriol 41:406-409

Michel C, Nougayrede P, Eldar A, Sochon, de Kinkelin P (1997) Vagococcus salmoninarum, a bacterium of pathological significance in rainbow trout (Oncorhynchus mykiss) farming. Dis Aquat Org 30:199-208

Perera RP, Johnson SK, Collins MD, Lewis DH (1994) Streptococcus iniae associated with mortality of Tilapia nilotica $x$ T. aurea hybrids. J Aquat Anim Health 6:335-340

Pier GB, Madin SH (1976) Streptococcus iniae sp. nov., a beta hemolytic streptococcus isolated from an Amazon freshwater dolphin, Inia geoffrensis. Int $\mathrm{J}$ Syst Bacteriol 26: $545-553$

Toranzo AE, Cutrin JM, Romalde L, Nunez S, Barja JL (1995) Antigenic characterization of Enterococcus strains pathogenic for turbot and their relationship with other Gram positive bacteria. Dis Aquat Org 21:187-191

Wallbanks S, Martinez-Murcia AJ, Fryer JL, Phillips BA, Collins MD (1990) 16S rRNA sequence determination for members of the genus Carnobacterium and related lactic acid bacteria and description of Vagococcus salmoninarum sp. nov. Int J Syst Bacteriol 40:224-230

Williams AM, Freyer JL, Collins MD (1990) Lactococcus piscium sp. nov. a new Lactococcus species from salmonid fish. FEMS Microbiol Lett 68:109-114

Zlotkin A, Eldar A, Ghittino C, Bercovier H (1998a) Identification of Lactococcus garvieae by PCR. J Clin Microbiol 36:983-985

Zlotkin A, Hershko H, Eldar A (1998b) Passible transmission of Streptococcus iniae from wild to cultured marine fish. Appl Env Microbiol 64:4065-4067

Submitted: May 14,1998; Accepted:December 31, 1998

Proofs received from author(s): May 14, 1999 